\section{Influence of pigskin on Salmonella contamination of pig carcasses and cutting lines in an Italian slaughterhouse}

\author{
Silvia Bonardi, ${ }^{1}$ llaria Bruini, \\ Irene Alpigiani, ${ }^{1}$ Alice Vismarra, ${ }^{1}$ \\ Elena Barilli, ${ }^{1}$ Franco Brindani, \\ Marina Morganti, ${ }^{2}$ Paola Bellotti, \\ Luca Bolzoni, 2 Stefano Pongolini² \\ ${ }^{1}$ Food Hygiene Unit, Department of \\ Veterinary Science, University of Parma, \\ Parma; ${ }^{2}$ Risk Analysis Unit, Institute for \\ Experimental Veterinary Medicine of \\ Lombardy and Emilia-Romagna, Parma, \\ Italy
}

\section{Abstract}

Ninety pig carcasses and twenty one food contact surfaces (FCSs) were tested for Salmonella in a slaughterhouse processing $c a .380 \mathrm{pigs} / \mathrm{h}$ between 2014-2015. Sampling was performed during seven sessions. Four carcass sites of 100 $\mathrm{cm}^{2}$ each (back, belly, jowl externally, and the diaphragmatic area internally) were swabbed after evisceration. Meat conveyors and dressing tables were tested swabbing areas of 200 to 400 $\mathrm{cm}^{2}$. After pre-enrichment in buffered peptone water, samples were tested by Salmonella MDS ${ }^{\circledR}$ assay and the presumptive positives were confirmed by the ISO 6579 method. Salmonella isolates were serotyped following the KauffmanWhite-Le Minor scheme and genotyped by $X b a \mathrm{I}$ pulsed field gel electrophoresis. Salmonella was isolated from 16/90 [17.8\%; confidence interval (CI) $95 \%=11.2-26.9]$ carcasses and 4/21 (19.0\%; CI $95 \%=7.7-40.0)$ FCSs. Four serovars were identified on carcasses. S. enterica 4,[5],12:i:was the most prevalent (43.75\%), followed by $S$. Rissen (31.25\%), $S$. Derby (12.5\%) and $S$. Bovismorbificans (12.5\%). Two serovars were found on FCSs, namely $S$. Derby (75\%) and $S$. Livingstone (25\%). During one sampling session, a failure in carcass dehairing occurred and caused significantly higher prevalence of carcass contamination (60\%) than in the remaining sessions. Moreover, in the same session, Salmonella prevalence was marginally significantly higher on FCSs than in the remaining sampling days, suggesting that dehairing affects contamination not only on carcasses, but also on the working surfaces.

\section{Introduction}

For many years Salmonella has been the second most common food-borne pathogen in the European Union after thermotolerant Campylobacter. In several food-borne outbreaks pork and pork products have been associated with strong epidemiological evidence to salmonellosis cases in humans; in 2013, for instance, $8.9 \%$ of 314 strong-evidence outbreaks were associated with the consumption of pig meat and products thereof. On the contrary, poultry meat and bovine meat were responsible for 5.1 and $1.6 \%$ of the strong-evidence salmonellosis outbreaks in the EU, respectively (EFSA and ECDC, 2015).

Pigs are often healthy carriers of different Salmonella serovars, with $S$. Typhimurium, $S$. Derby, $S$. enterica 4,[5],12:i:- (known as the monophasic variant of $S$. Typhimurium) and $S$. Rissen as the most prevalent (Arguello et al., 2012; EFSA and ECDC, 2015; Rostagno et al., 2007; van Hoek et al., 2012). These sub-clinically infected pigs may shed Salmonella with their faeces and/or keep the microorganisms in the gut, the associated lymph nodes or the tonsils (Fedorka-Cray et al., 1999), thus representing a potential contamination source during slaughtering operations and eventually a threat to human health.

The aim of this study was to investigate the prevalence of Salmonella on pig carcasses and the relationship between carcass contamination and cross-contamination of the cutting line in a slaughterhouse located in Northern Italy. Genotyping was performed to trace the distribution of the different strains between carcasses and the slaughter environment.

\section{Materials and Methods}

\section{Sampling}

From February 2014 to May 2015, 90 pig carcasses and 21 food contact surfaces (FCSs) at cutting section were tested for Salmonella in a slaughterhouse of Emilia Romagna region, Northern Italy. The abattoir had a capacity of ca. 380 pigs per hour. Pigs at slaughter in this area of the country are minimum nine-month old with an average live weight of $160 \mathrm{~kg}$, as required for the production of Parma ham, traditional salami and other cured pork products, which are Protected Designation of Origin (PD0) products. Carcasses belonged to 20 batches of pigs. A batch was defined as a group of pigs coming from a single herd on a given day. Pigs were reared in different herds of Northern Italy and were randomly sampled during 7 visits performed at intervals of 60-70 days. After evisceration three to seven carcasses per batch (average number: 4.5 per batch) were selected. The number of tested carcasses per visit varied among 10, 15 and 20. Four different carcass sites of $100 \mathrm{~cm}^{2}$ each (back, belly and jowl externally and the diaphragmatic area
Correspondence: Silvia Bonardi, Department of Veterinary Science, University of Parma, via del Taglio, 10, 43126, Parma, Italy.

Tel: + 39.0521.032744.

E-mail: silvia.bonardi@unipr.it

Key words: Salmonella enterica; Pig; Dehairing; Carcasses; Food contact surfaces.

Acknowledgments: the authors gratefully acknowledge Dr. Gisella Pizzin, Ms. Ida Poli and Ms. Giuseppina Trentadue (Department of Veterinary Science, University of Parma) for technical assistance.

Contributions: the authors contributed equally.

Conflict of interest: the authors declare no potential conflict of interest.

Funding: the study was funded by the University of Parma, Italy.

Received for publication: 30 November 2015 . Revision received: 16 March 2016.

Accepted for publication: 16 March 2016.

This work is licensed under a Creative Commons Attribution-NonCommercial 4.0 International License (CC BY-NC 4.0).

(C) Copyright S. Bonardi et al., 2016

Licensee PAGEPress, Italy

Italian Journal of Food Safety 2016; 5:5654

doi:10.4081/ijfs.2016.5654

internally) were swabbed before chilling using a sterile sponge moistened with buffered peptone water (BPW) (3M Sponge Stick; 3M Health Care, St. Paul, MN, USA). After sampling, the sponge was placed in a sterile bag and stored at refrigeration temperature.

Three environmental surfaces per visit were sampled for a total of 21 samples, represented by 10 dressing tables and 11 meat conveyors. The working surfaces were tested swabbing areas of different size (200 to $400 \mathrm{~cm}^{2} \mathrm{ca}$.) using a sterile sponge moistened with BPW (3M Health Care). Sponges were placed in sterile bags and immediately refrigerated. All samples were transferred to the laboratory under refrigeration on the day of collection and tested the same day.

\section{Salmonella detection and typing}

A total of 111 samples (90 carcass swabs and 21 environmental swabs) were tested using the molecular detection system assay for Salmonella (MDS Salmonella; 3M Health Care) based on the isothermal amplification and bioluminescence detection of specific sequences of Salmonella DNA. The samples were previously pre-enriched in BPW (3M Health Care) suspending each sponge in 100 $\mathrm{mL}$ of the liquid medium. The suspension was manually shaken for 2 min and then left at 
room temperature for $2 \mathrm{~h}$ for resuscitation. The cultures were incubated at $37 \pm 1^{\circ} \mathrm{C}$ for $18 \pm 2 \mathrm{~h}$. Thereafter, $20-\mu \mathrm{L}$ aliquots of the broth cultures were tested by the MDS Salmonella assay following the manufacturer's instructions. Positive results were considered presumptive positive to be confirmed by the ISO 6579:2002 reference method (IS0, 2002).

Following the ISO 6579:2002 (ISO, 2002) method, the BPW cultures were inoculated into $10 \mathrm{~mL}$ of Mueller-Kauffmann tetrathionate broth (MKTT; Oxoid, Basingstoke, UK) and 10 $\mathrm{mL}$ of Rappaport-Vassiliadis soy broth (RVSB; Oxoid), with dilution rations of 1:10 and 1:100, respectively. The MKTT and RVSB cultures were incubated at $37 \pm 1^{\circ} \mathrm{C}$ and $41.5 \pm 1^{\circ} \mathrm{C}$ for 24 $\mathrm{h}$, respectively. After incubation, a $10-\mu \mathrm{L}$ loop of each culture was streaked onto Xylose Lysine Desoxycholate agar (XLD; 0xoid) and Chromogenic Salmonella agar (Oxoid) plates, incubated at $37 \pm 1^{\circ} \mathrm{C}$ for $24 \mathrm{~h}$. Suspect colonies were seeded into Triple Sugar Iron Agar (Biolife, Milan, Italy), Lysine Iron Agar (Oxoid) and Christensen's Urea Agar (Biolife) and incubated at $37 \pm 1^{\circ} \mathrm{C}$ for $20-24 \mathrm{~h}$. Cultures showing reactions typical of Salmonella were tested by slide agglutination with an 0-omnivalent Salmonella serum (Denka Seiken, Tokyo, Japan). Biochemical identification to the genus level was performed by using the API $20 \mathrm{E}^{\circledR}$ microsubstrate system (BioMérieux, Marcy l'Etoile, France).

Salmonella serotyping was performed according to the White-Kauffmann-Le Minor scheme by slide agglutination with $\mathrm{O}$ and $\mathrm{H}$ antigen specific sera (Bio-Rad, Marnes-La Coquette, France; Denka Seiken; Sifin, Berlin, Germany). Definitive identification of S. enterica 4,[5],12:i:- and its differentiation from $S$. Typhimurium were obtained phenotypically by repeating phase inversion at least three times without evidencing expression of phase-two flagellar antigen and genotypically with the PCR protocol described by Barco et al. (2011).

Salmonella pulsed field gel electrophoresis (PFGE) typing was performed according to standard methods (PulseNet, 2010) with XbaI restriction of DNA. Confidence intervals in the observed prevalence were estimated by using the Wilson binomial approximation (Brown et al., 2001). Statistical significance of differences in prevalence between groups was evaluated through Fisher's exact tests.

\section{Results}

\section{Salmonella contamination in pig carcasses}

Salmonella was isolated from 16 out of 90 [17.8\%; confidence interval (CI) 95\%=11.226.9] carcasses in five out of seven visits. In these five visits, the prevalence of Salmonella- positive carcasses varied widely, ranging from $10 \%$ (in three visits) to 40 and $60 \%$ (in the remaining two visits). The observed differences in the contamination of pig carcasses among visits can be explained with specific conditions observed at the slaughterhouse on the sampling days.

During a sampling session a failure in the dehairing procedure of pig carcasses occurred and a total of nine carcasses out of $15(60 \%)$ ended up as Salmonella-positive. The prevalence on carcasses during this sampling session was significantly higher than in the remaining sessions (Fisher's exact test; $\mathrm{P}=9.3$ $10^{-7}$ ). In addition, carcasses coming from three different herds shared $S$. Bovismorbificans and S. enterica 4, [5], 12:i:- isolates belonging to the same PFGE genotype, suggesting contaminations between carcasses at the slaughterhouse. Conversely, during another sampling visit, 10 carcasses belonging to pigs coming from two herds were examined and Salmonella was isolated from four carcasses out of five belonging to a single herd, while all carcasses from the second herd were Salmonella-negative.

Four different Salmonella serovars were identified on pig carcasses (Table 1). S. enterica 4,[5],12:i:- was the most prevalent serovar (7/16; 43.75\%), followed by $S$. Rissen $(5 / 16$; $31.25 \%), S$. Derby $(2 / 16 ; 12.5 \%)$ and $S$. Bovismorbificans $(2 / 16 ; 12.5 \%)$. Salmonella isolates were genotyped by $X b a$ I PFGE and different profiles were found among $S$. enterica 4,[5],12:i:, $S$. Rissen and $S$. Derby serovars (Table 1). Two PFGE profiles were revealed for each serovar detected on pig carcasses.

\section{Salmonella contamination in the slaughterhouse environment}

Salmonella was detected in four out of 21 (19.0\%; CI 95\%=7.7-40.0) FCS samples (Table 1). Salmonella was isolated from $3 / 11$ (27.3\%) meat conveyors and 1/10 (10\%) dressing tables. Two serovars were identified, namely $S$. Derby $(3 / 4 ; 75.0 \%)$ and $S$. Livingstone (1/4; 25.0\%). $X b a$ I PFGE identified three different pulsotypes among $S$. Derby isolates. Salmonella on FCSs was isolated in three out of seven visits. Interestingly, two of the isolates were recovered during the sampling session when the failure in the dehairing procedure occurred. The prevalence in FCS samples during this sampling session was marginally significantly higher than in the remaining sessions (Fisher's exact test; $\mathrm{P}=0.0796$ ), suggesting that the dehairing procedure affects the contamination not only on carcasses, but also on the production surfaces in contact with them.

\section{Discussion}

The prevalence of carcass contamination by Salmonella in an Italian slaughterhouse processing $c a$. 380 pigs per hour was evaluated.

In our study the most common Salmonella serovars detected were $S$. enterica 4,[5],12:i:-, $S$. Rissen and $S$. Derby, as observed in previous surveys (Bonardi et al., 2013; Alpigiani et al., 2014). These serovars are commonly isolated from pigs in several EU member states and data reported by EFSA confirm the prevalence of $S$. Derby and $S$. enterica 4,[5],12:i:- in pigs at slaughter (EFSA, 2008; EFSA and ECDC, 2015). Moreover, in 2014, S. enterica 4,[5],12:i:- was responsible for more than $45 \%$ of the human cases of salmonellosis in Emilia Romagna region, Northern Italy. In that period, $S$. Derby ranked fifth among the serovars detected from affected humans, and $S$. Rissen was also included among the top 10 most common serovars. The detection of $S$. Bovismorficans on pig carcasses is interesting as this serovar caused a large outbreak in Germany due to the consumption of raw minced pork and fermented raw pork sausages (Zwiebelmettwurst) (Gilsdorf et al., 2005). In $2013 \mathrm{~S}$. Bovismorficans was responsible for $0.6 \%$ of all confirmed human cases of salmonellosis reported in the EU member states (EFSA and ECDC, 2015). These epidemiological and clinical data support the role of pigs as carriers of

Table 1. Salmonella serovars and number of pulsed field gel electrophoresis profiles detected on pig carcasses and food contact surfaces at cutting lines in an Italian slaughterhouse.

\begin{tabular}{lccc} 
Serovar & Carcasses (n) & FCSs (n) & PFGE profiles (n) \\
S. enterica 4,[5],12:i:- & $7(43.75)$ & - & 2 \\
S. Rissen & $5(31.25)$ & - & 2 \\
\hline S. Derby & $2(12.50)$ & $3(75.0)$ & 4 \\
S. Bovismorbificans & $2(12.50)$ & - & 1 \\
\hline S. Livingstone & - & $1(25.0)$ & 1 \\
Total & $16 / 90(17.8)$ & $4 / 21(19.1)$ & 10 \\
\hline
\end{tabular}

FCSs, food contact surfaces; PFGE, pulsed field gel electrophoresis. Values in brackets are expressed as percentage. 
Salmonella strains transmissible to humans.

A further important finding of the study was the significant increase in carcass contamination following a failure in the dehairing procedure that occurred during one of the sampling visits. Pigs were introduced into the slaughter line not properly dehaired and $60 \%$ of the tested carcasses ended up as Salmonella-positive, the highest contamination value of the study. This could have been the consequence of persistence of bristles on the pigskin.

Other studies support the role of pigskin in the microbiological contamination of pig carcasses. Rossel et al. (2009) observed that carcass contamination was directly linked to skin contamination of live pigs before stunning. Skin contamination rates were related with lairage duration and lairage hygienic conditions. In their study the probability of carcass surface contamination decreased from 59 to $35 \%$ whether the skin was contaminated or not. As polishing and singeing of pig carcasses during the slaughter process do not always overcome skin contamination by Salmonella, other post-harvest interventions could be suggested, such as a second singeing step after carcass polishing or decontamination of carcasses (De Busser et al., 2013). A second singeing could be particularly important because the polishing step seems to be responsible for $5-15 \%$ of all carcass contamination (Berends et al., 1997) due to the establishment of Salmonella on brushes and scrapes, which are difficult to clean (Borch et al., 1996). In addition, hot water decontamination at $80^{\circ} \mathrm{C}$ for 14-16 sec. and hot water decontamination followed by acid lactic are effective in reducing Salmonella on pig carcasses (Jensen and Christensen, 2000; Hamilton et al., 2010). Alternative treatments are represented by hand-held steam suction and the combination of steam and ultra-sound (Goldbach and Alban, 2006). Recently, regulation No 2015/1474/EC (European Commission, 2015) allowed food business operators (FBOs) to use recycled hot water to remove surface microbiological contamination from carcasses of domestic ungulates and farmed game under the precondition that the entire meat production chain strictly adheres to good manufacturing practices and hazard analysis critical control point principles. An important suggestion for FBOs can be the maintenance of high hygienic standards at lairage and the shortening of the lairage time, in order to reduce Salmonella spread among holding pigs belonging to the same batch (Bonardi et al., 2016).

A noteworthy component of hygiene control systems is the availability of rapid and reliable methods for the detection of Salmonella in food and environmental samples. The Salmonella MDS assay (3M Health Care) used in this survey was characterised by a rapid response time ( $24 \mathrm{~h}$ ) which allowed the FBO to quickly perform extraordinary interventions of cleaning and disinfections at the cutting line. Indeed, Salmonella cross-contamination from carcasses to the cutting equipment is considered unavoidable (Berends et al., 1998). In our study, Salmonella cross-contamination from carcasses to FCSs at the cutting line was revealed only on one sampling day, when the same $S$. Derby pulsotype was detected both on a carcass and a dressing table. In other sampling sessions, the pulsotypes found on the cutting equipment were not detected on the carcass surfaces. This result was probably linked to the small number of carcasses tested per day (10 to 20) in comparison with the large number of slaughtered pigs ( $c a .3000$ per day), which could transfer a variety of Salmonella strains onto the cutting line. Even if we did not find any recurring Salmonella strain on FCSs and therefore did not observe any persistent isolate on the cutting line, other authors demonstrated that the isolates found on the cutting equipment might be part of the resident flora, as Salmonella may persist in biofilms in the slaughterhouse environment (van Hoek et al., 2012; Piras et al., 2015).

Berends et al. (1998) demonstrated that when contaminated pig carcasses are routinely brought into cutting lines, interim cleaning and disinfection of surfaces and utensils during breaks and at the end of the working day would most likely prevent not more than about $10 \%$ of all cross-contamination that takes place during a working day.

\section{Conclusions}

Our study focused on carcass and FCS contamination in a large pig abattoir of Northern Italy, where $c a .3000$ pigs per day are processed. Carcasses contamination seemed to be influenced by hygiene practice during slaughter. This is the case of the failure in the dehairing of pigs, which was followed by the highest prevalence of Salmonella-positive carcasses ever seen in the study. The occasional failure in carcass dehairing demonstrated that also working surfaces were affected by an increment in Salmonella contamination. These findings are important because they confirm the role of bristles and pigskin in the final contamination of the carcasses and the slaughter environment. In addition, they stress the importance of hygienic measures during the whole slaughtering process, together with the implementation of any strategy to reduce the prevalence of Salmonella on the skin of pigs prior and during slaughter.

$S$. Derby, $S$. enterica 4,[5],12:i:-, $S$. Rissen and $S$. Bovismorbificans found on carcasses belong to serovars responsible for human foodborne infections linked to pig meat, thus con- firming the importance of reducing Salmonella contamination at slaughter. Since pork and products thereof are the type of meat most often linked to notified outbreaks of salmonellosis within the European Union, microbiological testing at slaughter represents a useful tool to monitor this food-borne infection.

\section{References}

Alpigiani I, Bacci C, Lanzoni E, Brindani F, Bonardi S, 2014. Salmonella enterica prevalence in finishing pigs at slaughter plants in Northern Italy. Ital J Food Safety 3:1609.

Arguello H, Carvajal A, Collazos JA, GarcíaFeliz C, Rubio P, 2012. Prevalence and serovars of Salmonella enterica on pig carcasses, slaughtered pigs and the environment of four Spanish slaughterhouses. Food Res Int 45:905-12.

Barco L, Lettini AA, Ramon E, Longo A, Saccardin C, Dalla Pozza MC, Ricci A, 2011. A rapid and sensitive method to identify and differentiate Salmonella enterica serotype Typhimurium and Salmonella enterica serotype 4,[5],12:i:by combining traditional serotyping and multiplex polymerase chain reaction. Foodborne Pathog Dis 8:741-3.

Berends BR, Van Knapen F, Mossel DA, Burt SA, Snijders JM, 1998. Salmonella spp. on pork at cutting plants and at retail level and the influence of particular risk factors. Int J Food Microbiol 44:207-17.

Berends BR, Van Knapen F, Snijders JM, Mossel DA, 1997. Identification and quantification of risk factors regarding Salmonella spp. on pork carcasses. Int $\mathrm{J}$ Food Microbiol 36:199-206.

Bonardi S, Alpigiani I, Bruini I, Barilli E, Brindani F, Morganti M, Cavallini P, Bolzoni L, Pongolini S, 2016. Detection of Salmonella enterica in pigs at slaughter and comparison with human isolates in Italy. Int J Food Microbiol 218:44-50.

Bonardi S, Bassi L, Brindani F, D'Incau M, Barco L, Carra E, Pongolini S, 2013. Prevalence, characterization and antimicrobial susceptibility of Salmonella enterica and Yersinia enterocolitica in pigs at slaughter in Italy. Int $\mathrm{J}$ Food Microbiol 163:248-57.

Borch E, Nesbakken T, Christensen H, 1996. Hazard identification in swine slaughter with respect of foodborne bacteria. Int $\mathrm{J}$ Food Microbiol 30:9-25.

Brown LD, Cat TT, DasGupta A, 2001. Interval estimation for a proportion. Stat Sci 16:101-33.

De Busser EV, De Zutter L, Dewulf J, Houf K, 
Maes D, 2013. Salmonella control in live pigs at slaughter. Vet J 196:20-7.

EFSA, 2008. Report of the task force on zoonoses data collection on the analysis of the baseline survey on the prevalence of Salmonella in slaughter pigs. Part A. EFSA $\mathrm{J}$ 135:1-111.

EFSA and ECDC, 2015. The European Union summary report on trends of zoonoses, zoonotic agents and food-borne outbreaks in 2013. EFSA J 13:3991.

European Commission, 2015. Regulation of 27 August 2015 concerning the use of recycled hot water to remove microbiological surface contamination from carcasses, 2015/1474/EC. In: Official Journal, L 225, 28 August 2015.

Fedorka-Cray PJ, Bailey JS, Stern NJ, Cox NA, Ladely SR, Musgrove M, 1999. Mucosal competitive exclusion to reduce Salmonella in swine. J Food Protect 62:1376-80.

Gilsdorf A, Jansen A, Alpers K, Dieckmann H, van Treeck U, Hauri AM, Fell G, Littmann M, Rautenberg P, Prager R, Rabsch W, Roggentin P, Schroeter A, Miko A, Bartelt E, Braunig J, Ammon A, 2005. A nationwide outbreak of Salmonella Bovismorbificans PT24, Germany,
December 2004-March 2005. Eurosurveillance 10:2667.

Goldbach S-G, Alban L, 2006. A cost-benefit analysis of Salmonella control strategies in Danish pork production. Prev Vet Med 77:1-14.

Hamilton D, Holds G, Lorimer M, Kiermeier A, Kidd C, Slade J, Pointon A, 2010. Slaughter floor decontamination of pork carcasses with hot water or acidified sodium chloride. A comparison in two Australian abattoirs. Zoonoses Public Health 57:16-22.

ISO, 2002. Microbiology of food and animal feeding stuffs. Horizontal method for the detection of Salmonella spp. ISO Norm 6579:2002. International Organization for Standardization, Geneva, Switzerland.

Jensen T, Christensen H, 2000. Decontamination of pork. Documentation of the effect through investigations in the abattoir. Danish Meat Research Institute, Taastrup, Denmark.

Piras F, Fois F, Consolati SG, Mazza R, Mazzette $\mathrm{R}, 2015$. Influence of temperature, source, and serotype on biofilm formation of Salmonella enterica isolates from pig slaughterhouses. J Food Protect 10:1875-8.

PulseNet, 2010. One-day (24-28 h) standardized laboratory protocol for molecular sub- typing of Escherichia coli 0157:H7, Salmonella serotypes, Shigella sonnei, and Shigella flexneri by Pulsed Field Gel Electrophoresis (PFGE). Available from: www.cdc.gov/pulsenet/protocols/ecoli_salmonella_shigella_protocols.pdf

Rossel R, Jouffe L, Belceil P-A, 2009. Analysis of factors associated with Salmonella isolation on pork carcass using bayesian networks. Journées Recherche Porcine 41:438.

Rostagno MH, Hurd HS, McKean JD, 2007. Salmonella enterica prevalence and serotype distribution in swine at slaughter. In: Proceedings of the Seventh International Safepork Symposium on the Epidemiology and Control of Foodborne Pathogens in Pork. Verona, Italy, May 9-11, pp 153-155.

van Hoek AH, de Jonge R, van Overbeek WM, Bouw E, Pielaat A, Smid JH, Malorny B, Junker E, Löfström C, Pedersen K, Aarts HJ, Heres L, 2012. A quantitative approach towards a better understanding of the dynamics of Salmonella spp. in a pork slaughter-line. Int J Food Microbiol 153:4552 . 